\section{El Homo sportivus en la formación de docentes de Educación Física en el Uruguay (1906-1956)}

\author{
Homo sportivus in Physical \\ Education Teachers' Training \\ in Uruguay (1906-1956)
}

\section{O Homo sportivus na formação de professores de Educação Física no Uruguai (1906-1956)}

\section{Resumen}

Esta investigación indaga sobre la configuración del deporte en Uruguay (1906-1956), especialmente sobre la formación de los docentes encargados de la enseñanza de la educación física. Como hipótesis central se plantea que el Homo sportivus se configuró a partir de la influencia anglosajona, a través del discurso batllista sobre el deporte y la llegada de los misioneros norteamericanos de la YMCA, en la segunda década del siglo Xx. En este periodo existieron distancias y desencuentros entre la práctica del deporte en las instituciones educativas y la práctica del fútbol y su expansión en la sociedad uruguaya.

\section{Palabras clave}

Deporte; formación docente; educación física; Uruguay

\section{Abstract}

This study inquires about the shaping of sports in Uruguay (1906-1956), especially about physical education teachers' training. The central hypothesis states that the Homo sportivus was configured following the anglo-saxon influence, through the batllista discourse about sports and the arrival of the YMCA American missionaries in the second decade of the twentieth century. By that time, there were differences and disagreements between the sport practice in educational institutions and the practice of soccer and its spreading in the Uruguayan society.

\section{Keywords}

Sport; teacher education; physical education; Uruguay

\section{Resumo}

Esta pesquisa questiona sobre a configuração do esporte no Uruguai (1906-1956), especialmente, sobre a formação dos docentes encarregados do ensino da educação física. Como hipótese central propõe-se que no Uruguai, o Homo sportivus se configurou pela influência anglosaxã, através do discurso batllista sobre o esporte e a chegada dos missioneiros norte-americanos da YMCA, na segunda década do século xx. Neste período existiram distâncias e desencontros entre a prática do esporte ao interior das instituições educativas e a prática do futebol e sua expansão na sociedade do Uruguai.

\section{Palavras-chave}

Esporte; formação de professores; educação física; Uruguai

Fecha de recepción: febrero 2 de 2016 Fecha de aprobación: marzo 25 de 2016

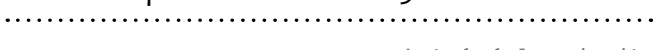

\section{Paola Dogliotti Moro*} nidades y Ciencias de la Educación (Fhuce-Udelar). Correo electrónico: paoladogliottimoro@gmail.com

Magíster en Enseñanza Universitaria por la Universidad de la República (Udelar), Uruguay. Doctoranda en Educación por la Universidad Nacional de La Plata (Argentina). Profesora adjunta del Instituto Superior de Edu-

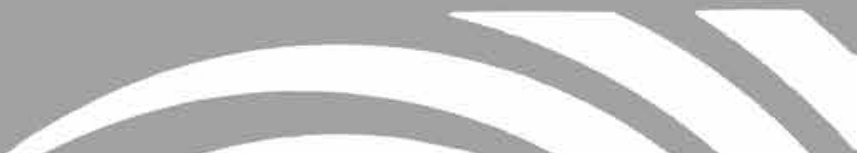




\section{Presentación ${ }^{1}$}

$\mathrm{E}$ ste trabajo tiene como propósito indagar sobre la configuración del deporte en el Uruguay, especialmente en la formación del personal especializado en educación física en el periodo comprendido entre la creación de la Comisión Nacional de Educación (CNEF) ${ }^{2}$ y hasta el año 1956, cuando se implementó un nuevo plan de estudios en el Instituto Superior de Educación Física (ISEF) ${ }^{3}$. Para esto se parte de un trabajo anterior (Dogliotti, 2012, 2015) en el cual se analizaron las discursividades en torno a la formación en educación física en el Uruguay entre los años 1874 y 1948. Se toman de esta investigación los principales hallazgos con relación a la configuración del deporte en esa formación y se intenta profundizar en los aspectos específicos del deporte y su relación con la gimnasia ${ }^{4}$, abarcando un lapso mayor de tiempo, hasta el año 1956, entendiendo que es en el período 1948-1956 donde se incluyen los deportes colectivos más importantes practicados en el país (fútbol y básquetbol) además del voleibol en el currículo de esta formación.

1 Este trabajo se inscribe en la línea de investigación "Políticas educativas, cuerpo y currículum" del Departamento de Educación Física y Prácticas Corporales que se enmarca en el Grupo de Políticas Educativas y Políticas de Investigación (GPEPI) del Instituto Superior de Educación Física (ISEF) de la Universidad de la República (Udelar), Uruguay.

2 Fundada el 7 de julio de 1911, por Decreto-Ley N. 3798 en el segundo gobierno del presidente José Batlle y Ordóñez para encargarse del desarrollo de la educación física tanto en el sistema educativo como fuera de este, en el todo el territorio nacional.

3 Desde 1939 y hasta el final del siglo xx, única institución en el Uruguay que otorgaba el título de "profesor de Educación Física" con reconocimiento oficial (Ministerio de Instrucción Pública). Existía una formación desde el año 1922 hasta la década de los 70, para secretarios técnicos y directores de Educación Física desarrollada en Montevideo en el marco del Instituto Técnico de la Federación Sudamericana de Asociaciones Cristianas de Jóvenes (ACJ), con una duración de cuatro años. Para un análisis de esta formación en sus primeras décadas, consultar Dogliotti $(2015,2012)$. El Curso de Profesores de Educación Física fue creado en el año 1939 en la órbita de la CNEF; en el año 1952 se le otorgó el nombre de ISEF (Gomensoro, 2012). Durante todo el siglo XX este dependió de la CNEF, que a su vez dependía a nivel ministerial de los diversos modos en los que se llamó el Ministerio de Educación y Cultura.

4 Para una investigación sobre la configuración del deporte y la gimnasia en los planes de estudio del ISEF entre los años 1939 y 1973 referirse a Torrón (2015).
Como hipótesis central del presente trabajo planteo que el Homo sportivus ${ }^{5}$ se configuró en el Uruguay fundamentalmente por la influencia anglosajona, a través del discurso batllista sobre el deporte y de la llegada de los misioneros de la Young Men Christian Association (YMCA) ${ }^{6}$ de Estados Unidos en la segunda década del siglo Xx. Para estos últimos, a diferencia de la gimnasia, el deporte desarrollaba de otro modo el espíritu patriótico, de equipo, las cualidades morales y tenía varios componentes motivacionales y atractivos para la juventud.

En el contexto mundial, han sido varias las discusiones entre la gimnasia y el deporte; en su génesis, a fines del siglo XVII, la gimnasia respondía a un cuerpo mecánico, como sistema de palancas, extraído del nuevo método científico de Galileo. Durante el siglo XIX comenzaron a emerger nuevos modelos en el campo de la física, que se trasladaron luego a la fisiología. Los impactos de la fisiología sobre los deportes y los juegos son claves; en ellos el cuerpo es identificado con la máquina, como productor y transformador de energía. Para el contexto francés, esta concepción, cuyo precursor fue Gustave Hirn (1815-1890), luego se trasladó en los estudios de Etienne-Jules Marey (1830-1904) con la colaboración de Georges Demenij. Ellos sentaron las bases para la representación del cuerpo como productor y transformador de energía. Fernard Lagrange (18461909), a partir de las investigaciones anteriores y con base en una concepción bioenergética del cuerpo, rechazó la gimnasia sueca estricta, con ejercicios basados en forma exclusiva en la fuerza, y promovió los juegos y deportes. A partir de estos cambios los ejercicios fueron clasificados ya no en función de la teoría mecánica en fáciles y difíciles (como en Amorós y Clias), sino en suaves, moderados y violentos (Gleyse, 2011).

Por otra parte, existió en el período considerado una serie de tensiones, distancias y desencuentros entre el Homo sportivus en las instituciones educativas (educación primaria, secundaria, terciaria: formación de profesores de educación física) y la expansión del fútbol en el Uruguay. Desde inicios de siglo xx el fútbol fue el deporte más practicado, sin embargo

5 Se toma esta expresión a partir del trabajo de Scharagrodsky (2011) utilizada para la gimnasia: "el Homo gymnasticus fue más que una metáfora de lo social. Fue la materialización de nuevos cuerpos y nuevas sensibilidades corporales, producto de complejas relaciones de poder-saber que no sólo produjeron sino que al mismo tiempo invisibilizaron, omitieron y excluyeron otras formas de ejercitarse, moverse, desplazarse, gesticular, sentir o experimentar la corporalidad" (Scharagrodsky, 2011, p. 20).

6 En español, ACJ. 
en las plazas de deporte ${ }^{7}$ copiadas del modelo de las YMCA de Estados Unidos no había espacio para este deporte. Las canchas eran pensadas para jugar voleibol y básquetbol, deportes creados en aquel país.

El trabajo se estructura en tres partes. En la primera se presentan las principales construcciones de sentido en torno al deporte en el proceso fundacional de la CNEF; entre las principales fuentes analizadas se destacan: la exposición de motivos de la ley de creación de la CNEF y de la revista Uruguay-Sport ${ }^{8}$, archivos de la CNEF. En la segunda parte se presentan los modos de significar al deporte por parte de la discursividad asociacionista, fundamentalmente, a través de su principal referente en Uruguay, el misionero Jess. T. Hopkins, radicado en el país entre 1912 y 1928, quién se hizo cargo a la vez del Departamento Físico de la Asociación Cristiana de Jóvenes en Montevideo y de la Dirección Técnica de la CNEF. Se analizarán aquí sus principales obras. En la tercera parte del trabajo se mostrará el lugar que ocupó el deporte y su relación con las restantes prácticas corporales, en los currículos de las modalidades de formación en el campo de la educación física a nivel estatal en el periodo seleccionado: en los cursos cortos de preparación de "maestros de educación física" desarrollados entre 1920 y 1936, y en la formación de profesores de educación física entre 1939 y 1956 , ambas modalidades en el ámbito de la CNEF. En esta última, como se explicitará, las cargas horarias destinadas a los deportes colectivos (fútbol, básquetbol y voleibol) frente a las gimnasias era mínima o inexistente. Recién en el año 1948 se incluyeron deportes colectivos como el básquetbol, el voleibol y el fútbol, siempre con cargas horarias menores a las de las gimnasias.

\section{El Homo sportivus en la creación de la CNEF}

Los términos juegos atléticos y deportes o sports muchas veces eran usados en forma indistinta en las primeras décadas del siglo $\mathrm{xx}$, tanto por políticos,

7 Las plazas de deportes se crearon en nuestro país a partir de la creación de la cnef. Fueron construidas con base en el proyecto de su primer presidente, Smith (1913), llamadas también "plazas vecinales de cultura física" o "plazas de juego populares". Eran centros populares de uso gratuito, tenían fines recreativos dirigidos a toda la población. En 1920, la cnef contaba con un total de 31 plazas de deportes, y proyectaba la construcción de 9 más. En el año 1923 había 41 plazas instaladas y 27 proyectadas (de las cuales 7 se hallaban en construcción) (CNEF, 1923, p. 157).

8 Editada mensualmente entre 1918 y 1926, fue difundida en varios países de América, entre los que se destacan: Argentina, Chile, Paraguay, Perú, Brasil y Estados Unidos. médicos higienistas y pedagogos como por los primeros misioneros de la YMCA que vinieron a radicarse a los países sudamericanos desde Estados Unidos.

En el discurso del presidente José Batlle y Ordóñez, cuando presentaba el proyecto de ley del poder ejecutivo para la creación de los juegos atléticos anuales el 7 de julio de $1906^{9}$, al igual que lo había hecho José Pedro Varela ${ }^{10}$ treinta años antes, se muestra cierta idealización de la "raza inglesa". Así, en palabras de un escritor francés, exaltaba sus "bellos" rasgos corporales adquiridos a través de la práctica del deporte:

Un escritor francés que había residido largo tiempo en Londres, decía a su regreso a Francia, la multitud le había parecido menos bella que la inglesa. Allá, agrega, el hombre es esbelto, fino, de una real elegancia, y esa superioridad parece responder a dos causas: alimentación más racional, y práctica diaria de los deportes.

Estimulemos, pues, los deportes, recordando que influyen eficazmente en la mayor salud del pueblo, y son además, una escuela de la voluntad y el ánimo. (CNEF, 1949, p. 4)

Se presentan, además de los beneficios eugenésicos del deporte y relativos a la salud, aquellos referidos al cultivo espiritual del "pueblo": la voluntad y el ánimo son dos ingredientes que no pueden faltar en la formación moral de cada ciudadano. Mediante los deportes se proponía un ideal de masculinidad marcado por atributos como la esbeltez y la elegancia del gentleman inglés. Batlle ${ }^{11}$ otorgaba gran importancia a los sports de origen anglosajón. Así: "La práctica de los deportes modernos partió de la colectividad británica. Su difusión en la sociedad uruguaya siguió la misma lógica que en Gran Bretaña y en otros países: desde las élites, en cascada hacia el resto de la población" (Luzuriaga, 2009, p. 53). Entre 1860 y 1890 se practicaba fútbol, remo, críquet y rugby en

9 Esta exposición es lo que dará lugar en el año 1911 a la promulgación de la ley de creación de la Comisión Nacional de Educación Física (CNEF) en la segunda presidencia de José Batlle y Ordóñez.

10 José Pedro Varela (1845-1879) es considerado "el padre" de la escuela pública uruguaya, laica, gratuita y obligatoria.

11 "El Estado batllista se sentía protagonista e intervenía en lo que entendía era su papel: apoyar a la comunidad en el sport" (Luzuriaga, 2009, p. 255). 
los colegios ${ }^{12}$ ingleses de Uruguay. El primero fue el predominante y comenzó a practicarse en diferentes clubes en la última década del siglo XIX:

En el último lustro del siglo xix el fútbol comenzó a desbordar a las élites. Cada vez era disfrutado por mayor cantidad de personas y surgían más equipos fuera del influjo de las instituciones británicas y alemanas. [...] En el Novecientos hay más de 80 clubes. (Luzuriaga, 2009, p. 72)

En el discurso de los legisladores integrantes de la Comisión de Fomento ${ }^{13}$ expresado en la exposición de motivos de la ley que crea la CNEF y los juegos atléticos, se muestra el poder democratizador que le otorgaban a la educación física fundamentalmente a través de los deportes. Al igual que la escuela para Varela, el deporte para los diputados tenía la virtud de igualar a los sujetos, nivelaba mucho más que la cultura intelectual y de este modo las "clases sociales" desaparecían. Se podría caracterizar este pensamiento, haciendo una traducción del optimismo pedagógico que caracterizó la constitución de los sistemas educativos latinoamericanos, como optimismo deportivo:

En la práctica de los sports, los hombres se acercan y se vinculan, las clases sociales desaparecen y se nivelan más, mucho más que por la cultura exclusivamente intelectual. Todo lo que la educación intelectual es capaz de aristocratizar el espíritu, la educación física lo democratiza. El intelectual puro tiende casi siempre a destacarse, a sobresalir, a distinguirse del resto del grupo [...] mientras que en los ejercicios físicos racionales el secreto del éxito reside más en la solidaridad del compañerismo. [...] La cultura intelectual [...] desarrolla el egoísmo necesario para la vida de la personalidad, y la cultura física [...] desarrolla el altruismo necesario para la vida social. (CNEF, 1949, p. 11)

En el modo de explicar los beneficios sociales que se obtienen del desarrollo de los sports, se presentaba una concepción de lo social cercana al funcionalismo durkheimiano: "el individuo humano, la célula social como la célula orgánica, no vive ni vale sino en la acción conjunta y solidaria con las demás células" (CNEF, 1949, p. 10). La sociedad era vista como un conjunto de órganos en equilibrio y "la gran fuerza educativa de los ejercicios físicos desde el punto de vista social radica esencialmente en el poderoso

12 Se entiende por colegio aquellas instituciones privadas dedicadas a la formación de nivel primario y secundario de enseñanza básica.

13 Antonio Cabral, Manuel B. Otero, Domingo Arena, Alberto F. Canesa, Victor B. Sudriers, Santiago Rivas. sentimiento de solidaridad que ellos despiertan" (CNEF, 1949, p. 11). La sociedad y los sports, lejos de ser vistos como conflicto de fuerzas opuestas, eran entendidos como en una solidaridad orgánica de cada uno de sus miembros o jugadores. Así se lograría la "marcha ascendente de la civilización [...] y el progreso" (CNEF, 1949, p. 10).

En las páginas de la revista Uruguay Sport, Delgado $^{14}$ recurría a los griegos antiguos, y exaltaba su belleza centrada en la fuerza corporal desarrollada a través de la práctica de los deportes ${ }^{15}$ :

Nadie puede decir lo que de sí daría un pueblo en donde el hábito de los ejercicios físicos fuera unánime. Todavía no ha sido sobrepasado el esplendor de la Grecia, el pueblo deportista por excelencia, y es necesario pensar que si es tan fuerte su belleza es porque nadie ha sabido comprender como él la belleza de ser fuerte. (Delgado, 1918, p. 6)

En los discursos de la época, el lugar otorgado a los deportes, incluido el atletismo, era mayor que el de otras prácticas, como la gimnasia:

La mejor escuela del carácter es el culto de los deportes. Todos los juegos atléticos están basados en el obstáculo y en la dificultad y cuanto más peligros haya que salvar o más resistencia que vencer, mayor será, es axiomático, la cantidad de energía que se necesita desplegar. El triunfo en materia de sport, no sonríe solamente a más fuerte [sic] desde el punto de vista físico, sino también desde el punto de vista moral. (Delgado, 1918, pp. 5-6)

A los atributos de fuerza desplegados por los sports se sumaban los de orden moral. Eran innumerables los discursos altruistas en relación al deporte como desarrollo de valores morales. Así,

En la divulgación del deporte hay algo más importante que su tecnicismo real, existe el despertar de una Moral cuyo desenvolvimiento es la más positiva contribución a la defensa de la humanidad [...] Dar al deporte no solo su valor educativo de nuestra
14 José M. Delgado (1884-1956): uruguayo, doctor en Medicina y diputado por el batllismo.

15 Desde nuestra perspectiva, los deportes son una práctica moderna (Elías y Dunning, 1995). Llamar deporte a las prácticas corporales de la antigüedad griega es perder la perspectiva histórica y mirar lo antiguo desde lo moderno. Esto fue una constante en muchos de los textos de historia de la educación física hasta mediados de siglo $\mathrm{xx}$, dentro de los que podemos citar a Blanco (1948) para el caso uruguayo. 
organización humana sino también la significación de su real predominio como escuela de Disciplina, de Moral y de Respeto. (Colombo ${ }^{16}, 1918$, p. 21)

En una conferencia dictada por el doctor Francisco Ghigliani ${ }^{17}$ en el Primer Congreso Nacional contra el Alcoholismo, titulada "Influencia de la cultura física en la lucha contra el alcoholismo", también se les adjudicaba gran importancia a los deportes y se recurría para esto a poner como modelo a los ingleses:

Se ve a infinidad de sportsmen que no beben alcohol o no fuman más, a raíz de un entrenamiento, y que no beben alcohol ni fuman -y es esto lo más importante-, porque tienen conciencia del mal que les hace.

El entrenamiento sportivo además, es una escuela de carácter, donde se aprende a dominarse a sí mismo para llegar a determinado fin. La lucha sportiva, no sólo durante el período previo de la preparación, sino aún durante el desarrollo de los juegos y torneos, obliga a un continuo ejercitamiento de la voluntad que es el rasgo fundamental del carácter. El respeto a las estipulaciones legales, la conciencia del valor del esfuerzo solidario, la confianza y la fé en el poder de la energía, el hábito de dominar el impulso naciente, la voluntad ejercitada en dominar el deseo, etc. hacen del sportman un hombre que dentro del valimiento intelectual, está en las mejores condiciones para actuar en el escenario de la vida. (Ghigliani, 1918b, pp. 335-336)

El deporte es la "escuela del carácter", voluntad, respeto de las reglas, esfuerzo, solidaridad, confianza, energía; el dominio de sí mediante el dominio de los

16 Esta cita es extraída del informe que el dr. Ángel Colombo presentó a la CNEF como Delegado Oficial en Francia e Italia. En él se detalla que "El Comité Olímpico Internacional representado por el Barón Pedro Coubertin, ha dispensado a nuestro país en la persona del Excmo. señor Ministro del Uruguay en París, Doctor Don Juan Carlos Blanco, y en mi carácter de Delegado Oficial de la CNEF la oportunidad de interesarse por nuestro desenvolvimiento sportivo [...] realzada en estos últimos tiempos por la brillante actuación de los sportivos uruguayos" (Colombo, 1918, p. 19).

17 Nació en Buenos Aires el 7 de junio de 1883, de joven se radicó con su familia en Montevideo. Fue doctor en medicina, dirigente de la Sociedad de Gimnasia y Esgrima L'Avenir, del Sporting Club del Uruguay y del Club Universitario del Uruguay. A partir de 1915 fue miembro de la CNEF, que lo nombró presidente de la Federación Deportiva Uruguaya hasta 1918 desde donde fundó y presidió las federaciones de básquetbol, atletismo, voleibol y natación. Fue diputado por el batllismo mientras siguió siendo el Secretario de la CNEF, desde donde se hizo cargo de la dirección de la revista Uruguay-Sport. En 1922 fue representante uruguayo del Comité Olímpico Internacional en los Juegos Latinoamericanos de Río, de 1922. A fines de ese año dejó su cargo en la CNEF (Gomensoro, 2004, pp. 5-7). deseos e impulsos eran todos atributos otorgados al deporte. Se establecía un cierto optimismo deportivo, se trataba al deporte con cierta esencialidad ${ }^{18}$, como si hubiera en su lógica constitutiva determinados valores morales. La vinculación del deporte con la ética no se caracteriza por una relación esencial o necesaria, sino más bien contingente (Aisenstein, Ganz y Perczyk, 2001, p. 186).

\section{E1 Homo sportivus en la discursividad asociacionista: Jess T. Hopkins}

Las contraposiciones entre la gimnasia y el deporte habían comenzado en Europa en la segunda mitad del siglo XIX. Los principales argumentos señalados provenían de la física y luego de sus impactos en la fisiología.

El cuerpo no era sólo una morfología "cerrada", simple arquitectura limitada a una disposición de palancas cuya clave se ha de hallar en la mecánica y la anatomía funcional (que ilustraría la gimnasia del siglo XIX), es un lugar de paso en el que se convierten energías cuya clave se encuentra en la termodinámica y una ciencia biológica que tiene en cuenta los trabajos de los químicos (lo cual ejemplificaría el entrenamiento deportivo). Los juegos, los deportes, se podrían legitimar de un modo distinto al de antes: los ejercicios dispersos y agitados (los deportes) cobraron importancia frente a los ejercicios esterotipados y disciplinados (gimnasia). (Holt y Vigarello, 2005, pp. 351-352)

Jess T. Hopkins ${ }^{19}$ recomendaba que se desarrollaran en el niño en forma predominante los "juegos atléticos" frente a la gimnasia: "no les demos ejercicios suecos, como creen muchos aficionados que deberíamos darle, pero démosle [sic] amplia oportunidad para moverse" (Hopkins, 1922, p. 3495). En este argumento Hopkins seguía las ideas de Gulik, director de Springfield College de la YMCA, donde Hopkins se graduó en Estados Unidos. “Gulik criticaba tanto la gimnasia simple y mecánica [...] creía

18 Por esencialismo entendemos la tendencia a caracterizar ciertos aspectos de la vida social si tuvieran una esencia o un núcleo (natural o cultural) fijo, inmutable (Goellner, 2008, pp. 138-139).

19 Jess T. Hopkins fue maestro de educación física por el Springfield College de la YMCA estadounidense. En 1912 se radicó en Uruguay para encargarse del Departamento de Educación Física de la Asociación Cristiana de Jóvenes (ACJ) de Montevideo, fundado en junio de 1912. Entre 1913 y 1918 ocupó el cargo de director técnico de la recién creada Comisión Nacional de Educación Física (CNEF). 
que el propósito de la educación física podía lograrse más acabadamente a través del deporte, al que consideraba 'natural' en su forma y ejecución" (Torres, 2011, p. 263) ${ }^{20}$. Gulik utilizó los argumentos de la psicología para fundamentar esta primacía deportiva. Él junto a Henry Curtis, un exalumno de Stanley Hall, "de activa participación en la YMCA, crearon en 1906 la Playground Association of America, cuyo objetivo era la creación de plazas de juegos y parques públicos" (Torres, 2011, p. 270). De aquí extrajo Hopkins el modelo de plazas de deportes desarrollado en Uruguay en la segunda década del siglo $\mathrm{xx}$, cuando ocupó el cargo de director técnico de la CNEF. Entre los principales argumentos a favor de los sports o juegos, Hopkins expresaba:

\begin{abstract}
La vida de juegos sanos y adecuados ha sido peculiarmente empobrecida en ese continente y es solamente en este último cuarto de siglo que los deportes se han hecho populares. Con más exactitud, se diría que el desarrollo intensivo y el interés en los juegos recién se ha manifestado dentro de los últimos diez años. Un juego nuevo dado a un pueblo, llega a formar parte de la herencia social de las generaciones futuras. Es algo que nadie puede quitarles después. Los programas de simple gimnasia son de incuestionable valor, pero fuera del beneficio directo que suministran al individuo que participa en ellos, no tienen ningún valor particular para las generaciones futuras. [...] nada dejarían a las nuevas generaciones siguientes fuera de los valores de salud obtenidos por los padres. En cambio, lo inverso ocurre con los juegos [...] siempre que se da un nuevo juego a un país se enriquece en proporción su herencia social. (Hopkins, 1927, pp. 29-30)
\end{abstract}

Se desprende de este testimonio el optimismo con que era presentado el deporte o "juegos" con relación al papel que desempeñaba en lo social y en la vida pública. Ubicaba, en contraposición, a la gimnasia como valor en términos domésticos y ligada al mejoramiento de la salud.

El movimiento escolanovista desarrollado en los Estados Unidos a través de educadores progresistas propiciaron el advenimiento de la "Nueva Educación Física” (Torres, 2011, p. 267) que ubicó en el centro la defensa de los juegos o deportes frente a la gimnasia. Esta última centrada en la disciplina, la repetición y el orden en contraposición a los deportes que contribuía a valorizar las diferencias individuales,

20 En Springfield College el profesor James Naismith creó el básquetbol, y el graduado William G. Morgan, el voleibol; deportes que fueron incorporados al programa de estudios y diseminados rápidamente por los misioneros que fundaron las ACJ en Sudamérica. el interés, el placer por el juego y la posibilidad de elección personal. Estos educadores se basaban en los principios desarrollados por las psicologías de G. Stanley Hall, Jhon Dewey, William James y Edward Thorndike (Torres, 2011, p. 267) ${ }^{21}$. De alguna manera podemos decir que en el Uruguay se dio un resultado exitoso de la doctrina Monroe que luchaba contra la colonización europea de las ideas en América Latina y promovía en cambio la estadounidense (Craviotto y Malán, 2014).

Hopkins recomendaba para la etapa de la niñez "juegos en grupo o cooperativos", ya que eran de su preferencia y "no solamente vigorizan y desarrollan su físico, sino que como un principio fundamental educan moralmente" (1918, pp. 28-29). Esta será quizás una de las razones por las cuales el deporte demoró tanto en ingresar a la escuela ${ }^{22}$. Para la etapa de la juventud prefería "los sports atléticos y juegos de competencia tan necesarios y fundamentales para el buen desarrollo físico y moral de nuestra adolescencia" (Hopkins, 1927, p. 33). En esta etapa el deporte actuaba como represión de los instintos sexuales: "la juventud en este período tiene muchas ideas extrañas y curiosas. El mejor remedio para combatir a éstas se administra por medio de juegos de acción y al aire libre" (Hopkins, 1918, p. 30). Estos desarrollaban "coordinación, sangre fría, agilidad, sacrificio, cooperación, valor y fuerza" y le permitían al joven "jugar con buenos y sanos compañeros. Una desviación en su camino puede conducirlo a ser un pervertido sexual, un criminal, o un ciudadano de poco valor para la sociedad" (Hopkins, p. 30). Es clave en su pensamiento el lugar puesto a lo sexual —algo que hay que reprimir - y la concepción de deporte como algo "bueno" en sí mismo; se presentaba una esencialización del deporte o lo que llamamos optimismo deportivo, como si hubiera en su lógica constitutiva determinados valores morales.

Pero también desarrollaba espíritu patriótico e idealizaba atributos como violencia, ingenio y energía:

en los países en donde se encuentran bien arraigados los juegos atléticos de competencia, se notará que ellos han dominado en un sentido el verdadero carácter de este país. Este carácter parece ser más violento, más ingenioso y más enérgico. Los térmi-

21 Para profundizar en las relaciones entre gimnasia y deporte en el Uruguay en este periodo, referirse a Craviotto y Malán (2014).

22 Además de las razones edilicias explicitadas en un trabajo anterior (Dogliotti, 2012, 2015). 
nos deportivos se han difundido en el idioma y la influencia moral del deporte ha sido, sin duda, un factor de mucho valor. (Hopkins, 1918, p. 33)

El deporte desarrollaba el "instinto de combate que está latente en todo joven de sangre roja"; lejos de verse con una lógica moderna, como parte de la sociedad capitalista, el deporte se presentaba originario de la antigüedad y en una continuidad de una lógica evolutiva etnológica.

Según el autor, se debía estimular a "las grandes masas de gente a tomar parte de [...] pruebas deportivas" (Hopkins, 1918, p. 35). A partir de los enunciados del Comité Olímpico Internacional que promovía el "deporte para todos", Hopkins señalaba que los juegos de competencia eran "proveedores de atletas y al mismo tiempo actividades de 'Juegos para todos'” (1927, p. 39). Con relación a la promoción de futuros juegos en el continente, expresaba que "no deben ser impuestos prematuramente en Sud América; ellos deben venir como resultado lógico de una participación amplia y general de los deportes [...] en sanos torneos deportivos" (1927, pp. 41-42). También señalaba que se debía promover la creación de federaciones deportivas, "con delegados elegidos con criterios amplios y sus deseos desinteresados de servicio [...] un gran paso hacia la eliminación de la política de partido" (1927, p. 46). Todo esto exigía "proclamar la excelencia del juego limpio, [...] normas elevadas de amateur [...] hábiles performances" (1927, p. 47). Se desprende de sus ideas cierto funcionalismo social, que no le permitía ver la lógica del deporte dentro de las pautas que impone el mercado. Podríamos decir que este optimismo deportivo estaba sustentado, en cierta medida, en una visión de la sociedad como un todo, con fuerzas solidarias e integradas al servicio del bien común.

En este sentido,

Toda la clase, todos los estudiantes de una institución, todos los soldados de una compañía y todas las compañías del regimiento juegan o toman parte en pruebas atléticas o ejercicios físicos organizados. ¡Qué día memorable será para la raza cuando se obtenga la realización de este ideal! (Hopkins, 1918, pp. 37-38)

Se desprende del pensamiento de Hopkins la importancia que le otorgaba al servicio militar como parte fundamental de una nación y el vínculo estrecho entre el gobierno estadounidense y la YMCA:

El Gobierno de los Estados Unidos de Norte América [sic] ha dado a esa Institución casi la exclusiva dirección del recreo de los soldados en sus campamen- tos. [...] El Dr. Mc Curdy ${ }^{23}$, actualmente el Director supremo de recreación del Ejército norteamericano en Francia, explicó el sistema de atletismo en masa: [...] Y en la guerra estos hombres prestan tantos servicios, en el momento del combate, como los mejores atletas. Estos serán más eficientes en sus deberes militares. [...] En un ataque, salvaremos las vidas de muchos si podemos mejorar la condición atlética del hombre común y corriente que encarna el soldado de hoy. Muchas veces este soldado ordinario que solo demuestra el cuarenta por ciento de eficiencia es el que impide un ataque o una acción ofensiva en la guerra. (Hopkins, 1918, pp. 39-40)

Los juegos atléticos o deportes, según Hopkins, eran una herramienta tanto educativa como militar, ambas al servicio de la nación.

En la primera Convención Continental de la Federación Sudamericana de Asociaciones Cristianas de Jóvenes realizada en Uruguay en 1914 se desarrollaron tres tareas centrales que debían estructurar al trabajo del "director" a cargo del "departamento de educación física”: “1. La enseñanza de juegos. 2. La promoción de la higienicultura; 3. La preparación de líderes" (Hopkins, 1927, p. 75). Por juegos se hacía referencia, además de los menos reglados, a todos los deportes. Esta tarea era destacada dentro de la YMCA a tal punto que el Comité Olímpico Internacional (COI) "ha colocado a los Directores de Educación Física de la Asociación, en varias partes del mundo, en posiciones estratégicas con respecto a la promoción de juegos regionales" (Hopkins, 1927, pp. 40-41). Por otra parte, en una de las resoluciones de la Segunda Convención Continental de 1919, se estableció que

\begin{abstract}
nuestra Asociación haga todo lo posible para crear o estimular la formación de federaciones deportivas modelos en aquellos países donde no existan, y que coopere con las federaciones nacionales existentes, teniendo en vista el propósito de formar eventualmente una gran federación continental en armonía con los últimos principios del deporte internacional organizado. (Hopkins, 1927, p. 46)
\end{abstract}

La predominancia de los deportes y su promoción tanto a nivel educativo como federado forma parte del movimiento asociacionista. Ellos crearon deportes como el voleibol y el básquetbol y han difundido y propagado muchos otros.

A partir del primer congreso, de 1914,

fue opinión unánime que ellos continuaran con el nombre en que son conocidos en la tierra de origen. Se propuso las "Basket Ball Rules of the Amateur Athletic Federation of Cook Country", como modelo 
para este Continente, y después de un prolongado debate y demostraciones, se las adoptó integralmente. (Hopkins, 1927, p. 73)

La homogeneización cultural (Puiggrós, 1990) ejercida por los países del norte, en este caso, Estados Unidos, fue clara en el continente. Podríamos hablar específicamente de homogeneización deportiva, como imposición de un modelo de deporte hegemónico y de sus reglas, lo que implicó la pérdida de otras formas de juegos y prácticas corporales identitarias de estas regiones.

\section{El Homo sportivus en la formación en el campo de la educación física}

En este apartado realizamos un análisis descriptivo de las relaciones entre el deporte y las restantes prácticas corporales en el currículo prescripto de la formación en educación física en el ámbito público (CNEF) en el periodo seleccionado para esta investigación (1906-1956).

A partir de Dogliotti (2015, p. 181), a continuación se presentan los contenidos de la "parte práctica" ${ }^{24}$ de los cursos cortos desarrollados por la CNEF entre los años 1920 y 1936 para la preparación de maestros de educación física.

Cuadro 1. Contenidos de la parte práctica de los cursos de Preparación de Maestros de Educación Física (1920-1936).

\begin{tabular}{|c|c|c|c|}
\hline 1ㅇ: 1920 & 3ㅇ: 1924 & $4^{\circ}: 1925$ & 6: 1936 \\
\hline $\begin{array}{l}55 \text { h totales } \\
30 \text { h práctica }\end{array}$ & $\begin{array}{l}84 \text { h totales } \\
59 \text { h práctica }\end{array}$ & $\begin{array}{l}70 \text { h totales } \\
50 \text { h práctica }\end{array}$ & $\begin{array}{l}984 \mathrm{~h} \text { totales } \\
432 \mathrm{~h} \text { práctica }\end{array}$ \\
\hline $\begin{array}{l}\text { Juegos para distintos } \\
\text { grupos: } 8 \mathrm{~h} \\
\end{array}$ & Juegos de conjunto $12 \mathrm{~h}$ & Juegos de conjunto & $\begin{array}{c}\text { Ejercicios de conjunto (al aire libre) } \\
\text { Juegos para plazas de deportes }\end{array}$ \\
\hline $\begin{array}{l}\text { Juegos en equipos } \\
8 \text { h Básquet Ball } \\
\text { Volley Ball } \\
\text { Base Ball } \\
\\
\text { Tennis } \\
\text { Varios }\end{array}$ & $\begin{array}{c}\text { Juegos organizados14 h } \\
\text { BasKet-Ball } \\
\text { Volley-Ball } \\
\text { Base-Ball (indoor y outdoor) } \\
\text { Captain-Ball } \\
\text { Newcomb } \\
\text { Balón } \\
\text { Hazena } \\
\text { Cage-Ball }\end{array}$ & $\begin{array}{l}\text { Juegos organizados: } \\
\text { ídem } 1924\end{array}$ & $\begin{array}{c}\text { Juegos organizados: } \\
\text { BasKet-Ball } \\
\text { Volley-Ball } \\
\text { Base-Ball (indoor y outdoor) } \\
\text { Captain-Ball } \\
\text { Newcomb } \\
\\
\text { Tennis, } \\
\text { Fied hockey } \\
\text { Football } \\
\end{array}$ \\
\hline Atletismo $6 \mathrm{~h}$ & Atletismo $8 \mathrm{~h}$ & Atletismo & Atletismo \\
\hline $\begin{array}{c}\text { Gimnasio (cerrado } \\
\text { y al aire libre): } 6 \mathrm{~h} \\
\text { Marchas } \\
\text { Mov. calisténicos } \\
\text { Aparatos }\end{array}$ & \begin{tabular}{|} 
Ejercicios de conjunto (10 \\
h) Formaciones gimnásticas, \\
marchas, carreras, etc. \\
Ejercicios calisténicos rítmicos, \\
por orden, con manubrios, \\
con barras, con clavas indias. \\
Danzas gimnásticas \\
y regionales \\
\end{tabular} & $\begin{array}{l}\text { Ejercicios de con- } \\
\text { junto: ídem. } 1924\end{array}$ & $\begin{array}{c}\text { Gimnasia pedagógica } \\
\text { Marchas y variaciones } \\
\text { Ejercicios calisténicos } \\
\text { Práctica de los diferentes sistemas } \\
\text { Ejercicios con aparatos de mano: } \\
\text { clavas, barras, manubrios, etc. } \\
\text { Danzas gimnásticas, } \\
\text { estéticas y regionales }\end{array}$ \\
\hline $\begin{array}{l}\text { Uso de aparatos en las } \\
\text { plazas de deportes: } 2 \mathrm{~h}\end{array}$ & $\begin{array}{c}\text { Ejercicios con los } \\
\text { aparatos pesados ( } 3 \mathrm{~h}) \\
\text { paralelas, caballo, barra } \\
\text { fija, burro, anillas }\end{array}$ & $\begin{array}{l}\text { Ejercicios con los } \\
\text { aparatos pesados: } \\
\text { ídem } 1924\end{array}$ & $\begin{array}{l}\text { Ejercicios con los aparatos } \\
\text { pesados: ídem } 1924\end{array}$ \\
\hline & Natación 12 h & Natación & Natación \\
\hline & & & Ejercicios de defensa personal \\
\hline
\end{tabular}

${ }^{*}$ Se conserva la ortografía de los documentos originales.

Fuente: Elaboración personal con base en CNEF (1920, 1924, 1925, 1930), en Dogliotti (2012, 2015, p. 181). 
Los tres campos de saberes de la educación física en los que se encontraban distribuidos los contenidos de los cursos eran:

- juegos: de conjunto o para distintos grupos

- gimnasias: marchas, ejercicios calisténicos, con aparatos de mano y pesados, danzas gimnásticas

- deportes: individuales (natación y atletismo) y colectivos (organizados o en equipo)

Como se desprende del cuadro 1, en ese momento los deportes eran los que ocupaban un espacio predominante en la formación de los maestros de Educación Física (más del doble de carga horaria que las gimnasias y los juegos). Los deportes fueron traídos de los Estados Unidos, a través de los profesores que se formaron en aquel país ${ }^{25}$; es claro en sus denominaciones que la tradición anglosajona primó sobre la latina. Es de destacar que el fútbol recién ingresa a los cursos impartidos en la CNEF en la década de los treinta. Si bien en el país su inclusión se dio fuera del sistema educativo público, ya hacía años que había sucedido; este deporte no se consideraba de valor pedagógico en la década de los veinte. Esto puede ser parte del pensamiento de la época; Vaz Ferreira ${ }^{26}$, en una conferencia ofrecida en el seno de la CNEF, en el año 1927, al comparar dos deportes de origen inglés - el golf y el fútbol- expresa que el segundo, a diferencia del primero:

25 Entre ellos se destacan: Jess Hopkins (estadounidense graduado en educación física en la Universidad de Springfield, Director técnico de la CNEF y de la ACJ Montevideo), Fred Dickens (estadounidense, graduado en Educación Física por la Universidad de Springfield, director de Educación Física de la ACJ de Buenos Aires), Henry Sims (estadounidense, graduado de Educación Física por el YMCA College de Chicago, director de Educación Física de la ACJ de Río de Janeiro), James Summers (estadounidense, graduado en Educación Física por la Universidad de Springfield, director de Educación Física del Instituto Técnico Sudamericano de las ACJ), Roy E. Thompson (director de la ACJ de Montevideo), y los siguientes discípulos de Hopkins en Montevideo que fueron a estudiar Educación Física a las universidades de la YMCA en Estados Unidos y luego ocuparon cargos importantes en la CNEF: Julio Rodríguez, Julio Pereyra, Hugo Grassi y Emilio Chiapella.

26 Carlos Vaz Ferreira (1872-1958) es considerado uno de los filósofos uruguayos más reconocidos en la primera mitad del siglo xx por su vasta obra en relación a temáticas filosóficas y su interés en problemáticas educacionales. Desde fines de siglo fue catedrático de Filosofía en la Universidad. Fue miembro del Consejo Directivo de Instrucción Primaria (1900-1915) y decano de Preparatorios de 1904 a 1906. Entre los años 1929 y 1943 ocupó con interrupciones el cargo de rector. Desde 1946 hasta su fallecimiento dirigió la Facultad de Humanidades y Ciencias, cuya creación impulsó desde el rectorado. es un juego de un valor sumamente discutible como elemento de educación física; interesantísimo espectacularmente [...] puesto que desarrolla en exceso unos miembros y no otros, suprime el ejercicio de los brazos, desarrolla desproporcionadamente las piernas, produce excesivo número de lesiones, gran cantidad de esos extraños inválidos, que no pueden dedicarse a otros ejercicios. (Vaz, 1959, p. 42)

La cita precedente es más que elocuente, el discurso de Vaz Ferreiria pudo haber sido importante en el seno de la CNEF para que el fútbol retardara su ingreso en las plazas de deportes y en los diversos niveles del sistema educativo.

A continuación, a partir de Dogliotti (2015, p. 218) se presentan las materias pertenecientes según nuestro criterio al área técnico profesional, su distribución en los tres años de estudio y la carga horaria del Curso de Profesores de Educación Física del año $1945^{27}$, curso que había sido creado en el año 1939 en el ámbito de la CNEF.

Cuadro 2. Materias del área técnico-profesional del Curso de Profesores de Educación Física del año 1945: listado de materias, distribución por año y cargas horarias.

\begin{tabular}{|c|c|c|c|c|}
\hline Asignaturas & $\begin{array}{c}\mathbf{1}^{\text {er }} \\
\text { año }\end{array}$ & $\begin{array}{c}\mathbf{2}^{\circ} \\
\text { año }\end{array}$ & $\begin{array}{c}3^{\text {er }} \\
\text { año }\end{array}$ & $\begin{array}{c}\text { Carga } \\
\text { horaria total }\end{array}$ \\
\hline & \multicolumn{5}{|l|}{} & $\mathbf{2 3 2 4}$ \\
\hline $\begin{array}{c}\text { Asignaturas } \\
\text { área técnico } \\
\text { profesional }\end{array}$ & \multicolumn{5}{|l|}{ Horas semanales } & 1522 \\
\hline Gimnasia & 3 & 4 & 4 & 320 \\
\hline Atletismo & 2 & 3 & 4 & 288 \\
\hline Natación & 2 & 2 & 2 & 192 \\
\hline Remo & 2 & 2 & 2 & 192 \\
\hline Esgrima & 2 & 2 & 2 & 192 \\
\hline Boxeo & & 2 & 2 & 128 \\
\hline Tenis & $1\left(2 .^{\circ}\right)^{28}$ & 1 & 2 & 112 \\
\hline $\begin{array}{c}\text { Juegos y } \\
\text { Deportes }\end{array}$ & 1 & 2 & & 98 \\
\hline
\end{tabular}

Fuente: elaboración personal a partir de CNEF (1945) en Dogliotti (2015, p. 218).

27 Se selecciona el programa de estudios de 1945 como el más representativo del periodo 1939-1947) ya que de las dos fuentes encontradas en la época (Rodríguez, 1939; CNEF, 1945) es el que más coincide con las asignaturas realmente cursadas por los estudiantes. Es de destacar que en ese periodo los programas de estudio tenían anualmente algunas variaciones debido a la escasa disponibilidad de docentes que las dictaran. Para un análisis detallado de los cambios de unidades curriculares entre 1939 y 1947 referirse a Torrón (2015, p. 59).

28 Corresponden $\left(1^{\circ}\right)$ y $\left(2^{\circ}\right)$ a primer y segundo semestre, respectivamente. 
El área técnico-profesional representa el 65,5\% de la carga horaria total del plan de estudios. Con relación a la proporción de las materias agrupadas en: juegos, gimnasias y deportes, los primeros son el gran ausente en el plan de estudios, las gimnasias ocupan el 21\% del área, y los deportes los restantes 79\%. Hay una gran proporción los deportes individuales y las luchas, en tanto que los deportes colectivos son casi inexistentes, con tan solo una unidad curricular (Juegos y Deportes) que representa el 6,4\% del área.

Se puede establecer, en cierta medida, un quiebre discursivo hacia el interior del Curso de Profesores en relación con la predominancia otorgada a los deportes en las décadas anteriores en el currículo de los Cursos de Preparación de Maestros de Educación Física, en los discursos de Jess T. Hopkins, de la revista Uruguay-Sport, y de los políticos que fundamentaron el proyecto de creación de la CNEF y que matrizaron el discurso gestacional de esa institución. El fútbol, deporte predominante en la vida cotidiana de los jóvenes uruguayos desde principios de siglo, no estaba incluido en el programa del año 1945. El básquetbol y el voleibol, deportes practicados en las plazas de deportes —traídos por la influencia que las universidades de las YMCA estadounidenses tuvieron en el Uruguay, a través, fundamentalmente, de la figura de Hopkins - no formaron parte del currículo en el periodo 1939-1947; al igual que el fútbol, recién se incluyeron como materias específicas, en el plan de $1948^{29}$.

Aunque en el periodo comprendido entre los años 1948 y 1956 se desarrollaron dos nuevos planes de estudio (1948 y 1956), seleccionamos para este trabajo el del año 1956 como el más representativo de los cambios del periodo para luego realizar la comparación con el programa de 1945, el más representativo del periodo anterior (1939-1947). El plan de estudios de 1948 presenta pequeños cambios con relación a los programas de estudio de los años precedentes (1939-1947): agrega Danzas para mujeres, Fútbol y Aparatos para varones, Basquet-Voleibol para ambos sexos, Dietética y Nutrición, Biotipología y Nociones de Música e Impostación (CNEF, 1948) ${ }^{30}$. A continuación se presentan las materias pertenecientes al área técnico profesional, su distribución en los tres años de estudio y la carga horaria del plan de estudios del año $1956^{31}$.

Cuadro 3. Plan de estudio de la Carrera en Educación Física del año 1956:

listado de materias, distribución por año y cargas horarias.

\begin{tabular}{|c|c|c|c|c|}
\hline Asignaturas & 1. ${ }^{\mathrm{er}}$ año & $2:^{\circ}$ año & 3. ${ }^{\text {er }}$ año & Carga horaria total \\
\hline & & & & 3179 \\
\hline Área técnico profesional & \multicolumn{3}{|c|}{ Horas semanales } & 1513 \\
\hline Teoría de la Gimnasia & & $2\left(1^{\circ}\right)\left(2^{\circ}\right)^{32}$ & $2\left(1^{\circ}\right)\left(2^{\circ}\right)$ & 136 \\
\hline Gimnasia Educativa (fem. y mas.) & $3\left(1^{\circ}\right)\left(2^{\circ}\right)$ & $3\left(1^{\circ}\right)\left(2^{\circ}\right)$ & $2\left(1^{\circ}\right)\left(2^{\circ}\right)$ & 272 \\
\hline Gimnasia en Aparatos (mas.) & $1\left(2^{\circ}\right)$ & $1\left(1^{\circ}\right)\left(2^{\circ}\right)$ & $1\left(1^{\circ}\right)\left(2^{\circ}\right)$ & 85 \\
\hline Gimnasia Rítmica (fem.) & & $2\left(1^{\circ}\right)\left(2^{\circ}\right)$ & $1\left(1^{\circ}\right)\left(2^{\circ}\right)$ & 102 \\
\hline Atletismo (fem. y mas.) & $2\left(1^{\circ}\right)\left(2^{\circ}\right)$ & $2\left(1^{\circ}\right)\left(2^{\circ}\right)$ & $2\left(1^{\circ}\right)\left(2^{\circ}\right)$ & 204 \\
\hline Práctica Docente en Atletismo & & & $1\left(2^{\circ}\right)$ & 17 \\
\hline $\begin{array}{l}\text { Natación Práctico } \\
\text { Teórico } \\
\end{array}$ & $\begin{array}{l}2\left(1^{\circ}\right)\left(2^{\circ}\right) \\
1\left(1^{\circ}\right)\left(2^{\circ}\right)\end{array}$ & $\begin{array}{c}2\left(1^{\circ}\right) 1\left(2^{\circ}\right) \\
1\left(2^{\circ}\right)\end{array}$ & $\begin{array}{c}2\left(1^{\circ}\right) 1\left(2^{\circ}\right) \\
1\left(2^{\circ}\right)\end{array}$ & $\begin{array}{c}170 \\
68\end{array}$ \\
\hline Práctica Docente en Natación & & & $2\left(1^{\circ}\right)$ & 34 \\
\hline Fútbol & $2\left(1^{\circ}\right) 1\left(2^{\circ}\right)$ & & & 51 \\
\hline Técnica y Didá. Basket-Ball y Volley-Ball & $2\left(2^{\circ}\right)$ & $2\left(1^{\circ}\right)\left(2^{\circ}\right)$ & $1\left(1^{\circ}\right)$ & 119 \\
\hline Remo & $2\left(1^{\circ}\right)\left(2^{\circ}\right)$ & & & 68 \\
\hline Tennis & & & $1\left(1^{\circ}\right)$ & 17 \\
\hline $\begin{array}{l}\text { Deportes de Defensa Personal (mas. / fem.) } \\
\text { / Lucha / Boxeo / Esgrima (fem. y mas.) }\end{array}$ & & $1\left(1^{\circ}\right)\left(2^{\circ}\right)$ & $1\left(1^{\circ}\right)\left(2^{\circ}\right)$ & 68 \\
\hline Ritmos y Danzas & & $1\left(1^{\circ}\right)\left(2^{\circ}\right)$ & $2\left(1^{\circ}\right)\left(2^{\circ}\right)$ & 102 \\
\hline
\end{tabular}

Fuente: elaboración personal a partir de ISEF (1956).

29 El primer programa de la materia Fútbol encontrado es del año 1952 y el de básquetbol y voleibol, denominado Técnica y Didáctica del año 1954, si bien se incluyó en el plan de 1948 por primera vez.

30 Un cuadro de las asignaturas y cargas horarias del Plan 1948 se encuentra en Torrón (2015, p. 70).

31 Plan de Estudios proyectado en el Seminario del Instituto Superior de Educación Física, realizado en enero de 1956.

32 Corresponden $\left(1^{\circ}\right)$ y $\left(2^{\circ}\right)$ a primer y segundo semestre, respectivamente. 
El área técnico-profesional representa el $48 \%$ de la carga horaria total del plan de estudios. Al compararla con el programa de 1945 , se observa que si bien el porcentaje total en carga horaria disminuye en un $18 \%$, la carga horaria se mantiene prácticamente igual (disminuye en un total de $9 \mathrm{~h}$ ); no obstante, hay una redistribución de cargas horarias y tipos de materias incluidas en esta área que da cuenta de un cambio de constitución de los saberes técnicos del campo. Esto se presenta en el próximo cuadro, en el que se muestra una comparación de los saberes del área técnico profesional entre el programa del $45 \mathrm{y}$ el plan del 56.

Cuadro 4. Comparación de los saberes del área técnico profesional entre el programa del 45 y el plan del 59.

\begin{tabular}{|c|c|c|}
\hline $\begin{array}{c}\text { Área } \\
\text { técnico-profesional }\end{array}$ & $\begin{array}{c}\text { Programa } \\
\mathbf{1 9 4 5}(\mathbf{1 5 2 2} \\
\mathbf{h} / \mathbf{6 5 , 5} \%)\end{array}$ & $\begin{array}{c}\text { Plan 1956 } \\
(\mathbf{1 5 1 3} \mathbf{h} / \mathbf{4 8} \%)\end{array}$ \\
\hline Deportes & $1202 \mathrm{~h} / 79 \%$ & $816 \mathrm{~h} / 54 \%$ \\
\hline Gimnasias & $320 \mathrm{~h} / 21 \%$ & $595 \mathrm{~h} / 39 \%$ \\
\hline Juegos & $0,00 \%$ & $0,00 \%$ \\
\hline Danzas & $0,00 \%$ & $102 \mathrm{~h} / 7 \%$ \\
\hline
\end{tabular}

Fuente: Elaboración personal a partir de CNEF (1945) e ISEF (1956).
La gimnasia en su globalidad se diversifica y aumenta muchísimo su carga horaria al pasar de $320 \mathrm{~h}$ en el programa de 1945 a 595 h en este plan, que representa el $39 \%$ del área técnico profesional frente al $21 \%$ del plan de 1945 . Se crean materias como Teoría de la Gimnasia (1946), Gimnasia en Aparatos (para varones) incluida en 1948 y Gimnasia Rítmica (para mujeres). La materia Gimnasia cambia su nombre a Gimnasia Educativa diferenciada en Femenina y Masculina.

Los deportes representan el $54 \%$ del total del área técnico-profesional frente al $79 \%$ del programa del 45, su carga horaria baja en 386 h, debido al aumento de las gimnasias y al ingreso de Ritmos y Danzas.

En el cuadro 5 se compara la distribución de los tipos de deportes entre el programa del 45 y el plan del 56.

A nivel de los deportes individuales, Atletismo baja su carga horaria de $288 \mathrm{~h}$ a 221 h que incluyen la Práctica Docente en Atletismo como nueva asignatura. Por el contrario, Natación aumenta su carga horaria en $80 \mathrm{~h}$, en las cuales se incluye como novedad $34 \mathrm{~h}$ de Práctica Docente en esta disciplina.

Cuadro 5. Comparación de los tipos de deportes entre el programa de 1945 y el plan de 1956.

\begin{tabular}{|c|c|c|}
\hline Tipos de deportes & Programa 1945 (1202 h/79\%) & Plan 1956 (816 h / 54\%) \\
\hline Deportes individuales & $\begin{array}{c}672 \mathrm{~h} / 56 \% \text { (Atletismo, } \\
\text { Natación y Remo) }\end{array}$ & $\begin{array}{c}561 \mathrm{~h} / 69 \% \text { (Atletismo, } \\
\text { Natación y Remo) }\end{array}$ \\
\hline Colectivos & $98 \mathrm{~h} / 8 \%$ (Juegos y Deportes) & $\begin{array}{c}170 \mathrm{~h} / 21 \% \text { (Fútbol, } \\
\text { Basquet y Voley) }\end{array}$ \\
\hline Luchas & $320 \mathrm{~h} / 27 \%$ (Esgrima y Boxeo) & $\begin{array}{c}68 \mathrm{~h} / 8 \% \text { (Deportes de } \\
\text { Defensa Personal) }\end{array}$ \\
\hline Tenis & $112 \mathrm{~h} / 9 \%$ & $17 \mathrm{~h} / 2 \%$ \\
\hline
\end{tabular}

Fuente: Elaboración personal a partir de CNEF (1945) e ISEF (1956).

En el periodo 1948-1956 aparecen por primera vez los deportes colectivos: Fútbol con $51 \mathrm{~h}$ (se incluye por primera vez en el plan del 48 con $66 \mathrm{~h}$ ), Técnica y Didáctica del Basket-Ball y Volley-Ball con $119 \mathrm{~h}$ (se incluye por primera vez en el plan del 48 con $66 \mathrm{~h}$ ), pero Juegos y Deportes baja de $98 \mathrm{~h}$ a $51 \mathrm{~h}$ su carga horaria (en el plan del 48 su denominación era Juegos Pedagógicos, con una carga horaria de 88 h). Por otra parte, bajan en gran medida las cargas horarias de Remo de 192 a 68 h; Tennis, de 112 a $17 \mathrm{~h}$, y Esgrima con $192 \mathrm{~h}$ y Boxeo con $128 \mathrm{~h}$ se compactan en una única materia denominada Deportes de Defensa Personal (Masculina y Femenina) con tan solo $68 \mathrm{~h}$. Por otra parte, se agrega una nueva materia: Ritmos y Danzas, con $102 \mathrm{~h}$.

La identidad de las prácticas corporales en el periodo 1948-1956 se inscribe en la diversificación y profundización de las gimnasias y comienzan a emerger los deportes colectivos (Fútbol, Básquetbol y Voleibol) y el ritmo y la danza; por otra parte, prácticamente desaparecen las luchas de la malla curricular. El área del tiempo libre, el ocio y la recreación continúa como gran ausente en este periodo. 


\section{Consideraciones finales}

A partir de lo desarrollado a lo largo de este trabajo y de Dogliotti $(2012,2015)$ podemos decir que en el proceso gestacional de la CNEF se configuró cierto quiebre discursivo en relación con los modos de entender las prácticas corporales por parte de sus principales propulsores en el ámbito educativo estatal. Como habíamos sostenido en Dogliotti $(2012,2015)$ hubo un lento y paulatino pasaje de la gimnástica al sport. A través de lo desarrollado en la primera parte de este trabajo el deporte en la CNEF se configuró como el mejor mecanismo eugenésico tanto en términos espirituales como biológicos. Los discursos altruistas en relación con el deporte son más que abundantes tanto de los políticos como de los técnicos del campo específico de la educación física. En este entramado discursivo se configuró lo que llamamos un optimismo deportivo, que le otorgaba al deporte el poder de igualar a los sujetos, de hacer "desaparecer" las "clases sociales" dentro de lo social entendido como un órgano en equilibrio y al deporte como un instrumento esencial en la construcción de ese estado de solidaridad orgánica que contribuye a su estabilización.

De los documentos analizados podemos decir que hubo escasos o nulos elementos que contribuyeran a una reinterpretación local del Homo sportivus; en contraposición a esto, a través fundamentalmente de la discursividad asociacionista se configuró lo que llamamos en Dogliotti $(2012,2015)$ un movimiento de homogeneización deportiva, por el cual se adjudicaron a los deportes las mismas reglas, nombres y modos de juego que en los países anglosajones. $\mathrm{Si}$ bien sabemos que el fútbol fue el deporte que más respondió a estos procesos de Homogeneización deportiva, traído a través de los ingleses y el más practicado desde principios de siglo en el Uruguay, este no tuvo impacto directo en las formaciones analizadas ni en los discursos de sus referentes. Pareciera que hubiera habido un cierto divorcio o distancia entre las prácticas corporales desarrolladas dentro del sistema educativo y en la formación para dirigir las plazas de deportes y el ISEF, y las diversas asociaciones y federaciones deportivas (todas instituciones dependientes de la CNEF). Los modelos de construcción de las plazas de deportes fueron traídos de Estados Unidos y no tenían previsto en su diseño canchas de fútbol. El discurso vazferreiriano que no percibía a este deporte como medio educativo y de salud por llegar a excesos de fuerza y a configurar un "recordismo especialista" (Dogliotti, 2015) quizás haya sido uno de los factores determinantes para su lenta inclusión en el currículo de la formación de profesores de educación física.
Las discusiones entre la gimnasia y el deporte como medios de la educación física adquirieron mayor relevancia a través de la peculiar configuración de la discursividad asociacionista en el Uruguay. Fueron esta y el discurso batllista sobre el deporte los que introdujeron en el país, a través fundamentalmente de Jess T. Hopkins la preferencia de los sports frente a la gimnasia. Este argumentaba contundentemente las ventajas de los primeros en contraposición a la gimnasia, principalmente de origen sueco, no tanto apoyándose en los principios de la psicología escolanovista sino en argumentos de tipo altruista, social y patriótico. Sin embargo, una década después Vaz Ferreira sí introdujo esta discusión basándose en los principios fundamentales de las psicologías del momento. Podríamos conjeturar que la gimnasia de tipo sueco estuvo más cercana a los mecanismos de disciplinamiento de los cuerpos y que los deportes que introdujeron un modo "más libre" de ejercitación corporal, menos regulado externamente, con mayor disposición para la elección personal y el desarrollo de un cuerpo "más activo" estuvieron más cercanos a la regulación de las poblaciones y al advenimiento de un poder biopolítico (Foucault, 1992).

En el recorrido analizado sobre la formación del personal especializado en educación física en el ámbito público, podemos establecer tres grandes momentos en el tratamiento de los deportes colectivos y su relación con la gimnasia. Un primer momento comprendido entre los años 1920 y 1936, de formación de maestros de educación física, con gran afluencia del movimiento asociacionista, con cursos de corta duración, en los que se desarrollaba de un modo muy general una serie de deportes colectivos como el básquetbol, el voleibol y el béisbol desde inicios de la década hasta la inclusión tardía del fútbol en 1936. La importancia dada al deporte es mucho mayor que a la gimnasia, tanto en los discursos de sus formadores (Hopkins, Rodríguez, entre otros) como por la carga horaria destinada (duplicaba los deportes a las gimnasias). Un segundo momento entre los años 1939 y 1947, de inicio de la formación de profesor de educación física, única con reconocimiento oficial, de tres años de duración, donde no se presentaba la enseñanza de los deportes colectivos. Si bien la influencia del asociacionismo es menor al momento anterior, igual continúa, y los deportes individuales y las luchas tienen predominancia frente a las gimnasias. Un tercer momento, entre los años 1948 y 1956, donde se incluye la enseñanza de fútbol, básquetbol y voleibol pero con una carga horaria muy inferior a los deportes individuales como la natación y el atletismo. En ese momento, de comienzo del langladismo, a través de la influencia del profesor 
Alberto Langlade ${ }^{33}$, cuando asumió la secretaría del curso de profesores en el año 1948 y en 1949 la jefatura de estudios, fueron las gimnasias las que adquirieron mayor relevancia que los deportes ${ }^{34}$.

\section{Referencias}

Aisenstein, Á.; Ganz, N. y Perczyk, J. (2001). El deporte en la escuela. Los límites de la recontextualización. En Á. Aisenstein et al. (comps.). Estudios sobre deporte (pp. 167-198). Buenos Aires: Libros del Rojas.

Blanco, R. (1948). Educación Física. Un panorama de su historia. Montevideo: Impresora Adroher.

Colombo, A. (1918, febrero). Informe del Dr. Ángel Colombo. Uruguay-Sport. Archivos de la Comisión Nacional de Educación Física, 2, 18-21.

Comisión Nacional de Educación Física. (1920). Programa del Primer Curso intensivo Teórico-Práctico para Maestros de Plazas de Deportes. 2 al 12 de marzo de 1920. Montevideo: CNEF.

Comisión Nacional de Educación Física. (1923). Plan de Acción de la CNEF y conclusiones que se derivan del mismo. Presentado por su director técnico, sr. Julio J. Rodríguez. Montevideo: CNEF.

Comisión Nacional de Educación Física. (1924). Informes, horarios y otros detalles del Tercer Curso Intensivo Teórico-Práctico para la Preparación de Maestros de Educación Física a realizarse en Piriápolis del 17 al 29 de marzo de 1924. Montevideo: CNEF.

33 De destacada actuación en el ISEF, en 1946 ingresó como ayudante de Gimnasia Práctica y Teoría de la Gimnasia a cargo del profesor Pedro de Hegedus. Tras su retiro en 1947, Langlade asumió la titularidad desde 1948 hasta 1967. Con un gran reconocimiento a nivel nacional e internacional debido a su obra en el campo de la gimnasia, tuvo una destacada trayectoria en el país en la preparación física de selecciones de básquetbol y fútbol, y en la dirección técnica de cuadros reconocidos en estos deportes. Entre 1952 y 1967 fue profesor de Gimnasia Especial en la carrera de Fisioterapia de la Facultad de Medicina. En 1963 fue designado profesor extraordinario de la Facultad de Filosofía y Educación de la Universidad de Chile. Ha dictado cursos de posgrado y conferencias sobre las temáticas de la gimnasia y el entrenamiento deportivo a nivel internacional. El ISEF lleva su nombre a partir de la Ley 16086 del 18 de octubre de 1989

34 Según Torrón (2015, p. 181), en contraposición al deporte, "vemos en la gimnasia, al menos desde que Langlade asume la cátedra, una propuesta de corte más experimental y de indagación corporal. De acuerdo a la información relevada a través de las distintas fuentes, entendemos que en la gimnasia hubo un acercamiento al saber disciplinar, la gimnasia entendida como objeto teórico, donde su estudio y análisis definían las prácticas específicas". Para profundizar en las relaciones entre la gimnasia y el deporte en todo el periodo del langladismo referirse a Torrón (2015).
Comisión Nacional de Educación Física. (1925). Informes, horarios y otros detalles del Cuarto Curso Intensivo Teórico-Práctico para la Preparación y Perfeccionamiento de Maestros de Educación Física a Realizarse en el Balneario Atlántida del 23 de marzo al 2 de abril de 1925. Montevideo: CNEF.

Comisión Nacional de Educación Física. (1930). Educación Física en el Uruguay, por Julio J. Rodríguez, Director Técnico General de la CNEF. Publicación costeada por la Comisión Nacional del Centenario 1830-1930. (Apartado del "Boletín del Instituto Internacional Americano de Protección a la Infancia”). Montevideo: CNEF.

Comisión Nacional de Educación Física. (1948). Memorándum sobre la organización de los estudios y catedráticos del Curso para la Preparación de Profesores de Educación Física. Montevideo: CNEF.

Comisión Nacional de Educación Física. (1949). Exposición de Motivos para la ley que crea la CNEF y los juegos atléticos de la Comisión de Fomento de la honorable Cámara de Representantes del 13 de noviembre de 1906. En Comisión Nacional de Educación Física. La educación física y la recreación en el Uruguay. Origen. Plan de acción (pp. 6-13). Montevideo: CNEF.

Craviotto, A. y Malán, P. (2014). Entre la gimnasia y el deporte: la configuración de la educación física en el Uruguay (1911-1930). Políticas Educativas, 7 (1), $32-42$.

Delgado, J. (1918). Alrededor de la cultura física. UruguaySport. Archivos de la Comisión Nacional de Educación Física, 2, 3-6.

Dogliotti, P. (2012). Cuerpo y curriculum: discursividades en torno a la formación de docentes de educación física en Uruguay (1874-1948) (Tesis de maestría en Enseñanza Universitaria). Universidad de la República, Montevideo, Uruguay. Recuperado de http://posgrados.cse.edu.uy/sites/posgrados.cse.edu.uy/files/ tesis_paola_dogliotti.pdf

Dogliotti, P. (2015). Educación del cuerpo y discursividades en torno a la formación en educación física en Uruguay (1874-1948). Montevideo: Comisión Sectorial de Investigación Científica (CSIC) de la Universidad de la República (Udelar).

Elias, N. y Dunning, E. (1995). Deporte y ocio en el proceso de la civilización. México: Fondo de Cultura Económica.

Foucault, M. (1992). Historia de la sexualidad. Tomo 1. La voluntad de saber. Madrid: Siglo XxI.

Ghigliani, F. (1918a). Apuntes relacionados con los maestros de las plazas de deportes VI a XI. Uruguay-Sport. Archivos de la Comisión Nacional de Educación Física, 2, 7-12. 
Ghigliani, F. (1918b). La influencia de la cultura física en la lucha contra el alcoholismo. Uruguay-Sport. Archivos de la Comisión Nacional de Educación Física, 6, Montevideo, 333-343.

Gleyse, J. (2011). La metáfora del cuerpo máquina en la educación física en Francia. En P. Scharagrodsky (comp.). (2011). La invención del "Homo gymnasticus". Fragmentos históricos sobre la educación de los cuerpos en movimiento en Occidente (pp. 77-95). Buenos Aires: Prometeo.

Goellner, S. (2008). El deporte y la cultura fitness como espacios de generificación de los cuerpos. En P. Scharagrodsky (comp.). Gobernar es ejercitar. Fragmentos históricos de la educación física en Iberoamérica (pp. 137-152). Buenos Aires: Prometeo.

Gomensoro, A. (2004). El borrascoso nacimiento del Comité Olímpico Uruguayo. Revista isef digital 2, 1-17. Disponible en www.isef.edu.uy.

Gomensoro, A. (2012). Historia de la formación de docentes en educación física en Uruguay. En M. González (comp.). La educación física en Lantinoamérica. Orígenes y trayectorias de la formación de profesores (pp. 69-90). Tucumán: Edunt.

Holt, R. y Vigarello, G. (2005). El cuerpo cultivado: gimnastas y deportistas en el siglo XIX. En A. Corbin, J. Courtaine, G. Vigarello (dir.). Historia del cuerpo (tomo 2) (pp. 295-354). De la Revolución francesa a la Gran Guerra. Buenos Aires: Taurus.

Hopkins, J. (1918). A monograph on physical education written in Spanish. Graduation (tesis doctoral). International Young Men's Christian Asosiation College Springfield, Massachusetts.

Hopkins, J. (1922). Educación Física. Bases biológicas de los juegos atléticos. Uruguay-Sport. Archivos de la Comisión Nacional de Educación Física, 52, 3493-3498.

Hopkins, J. (1927). Quince años de educación física en las asociaciones de América del Sur (1911-1926). Montevideo-Buenos Aires: Mundo Nuevo.
Hopkins, J. (1956). Plan de estudios del año 1956. Asignaturas discriminadas por año, carga horaria y programas de cada una de las unidades curriculares. Montevideo: ISEF-CNEF. 8/07/1959.

Instituto Superior de Educación Física (ISEF). (1945). Curso para profesores. Periodo de inscripciones de ingresos para 1945. Montevideo: Curso para la Preparación de Profesores de Educación Física. CNEF.

Luzuriaga, J. (2009). El football del novecientos. Orígenes y desarrollo del fútbol en el Uruguay (1875-1915). Uruguay: Santillana.

Puiggrós, A. (1990). Sujetos, disciplina y curriculum en los orígenes del sistema educativo argentino. Buenos Aires: Galerna.

Rodríguez, J. (1939). Programa de Estudios. Instituto Nacional de Educación Física. Montevideo: CNEF.

Scharagrodsky, P. (2011). Presentación. En P. Scharagrodsky. (comp.). La invención del "Homo gymnasticus". Fragmentos históricos sobre la educación de los cuerpos en movimiento en Occidente (pp. 15-20). Buenos Aires: Prometeo.

Torres, C. (2011). La educación física en Estados Unidos. En P. Scharagrodsky, (comp.). La invención del "Homo gymnasticus". Fragmentos históricos sobre la educación de los cuerpos en movimiento en Occidente (pp. 253-277). Buenos Aires: Prometeo.

Torrón, A. (2015). Gimnasia y Deporte en el Instituto Superior de Educación Física (1939-1973): su configuración y su enseñanza. Tesis de Maestría en Enseñanza Universitaria. Montevideo: Comisión Sectorial de Enseñanza. Área Social. Universidad de la República. 194 p.

Vaz, C. (1959). Sobre Educación Física en los parques escolares (1927). Anales de Educación Física, 3 (1). Uruguay: CNEF. 